\section{GW23-e1139 EXPERIMENTAL RESEARCH ABOUT EFFECT OF CELL- CELL CONTACT TO MESENCHYMAL STEM CELLS DIFFERENTIATE INTO VASCULAR ENDOTHELIAL CELLS}

doi:10.1136/heartjnl-2012-302920a.7

\footnotetext{
${ }^{1}$ Peng Jin, ${ }^{2}$ Chi Luxiang. ${ }^{1} 187$ th Hospital of Chinese People's Liberation Army;

${ }^{2}$ Southwest Hospital, Third Military Medical University
}

Objectives Use the method of HUVECs were cultured with human mesenchymal stem cells by cell-cell contact to simulate intravascular environment after angioplasty and stent implantation, and research the role of cell-cell contact to induce MSCs differentiate into endothelial cells. The research provides the experimental basis for MSCs repair the damage to vascular endothelium and prevention of vascular restenosis after intervention.

\section{Methods}

1. We mixed HUVECs and MSCs by the same density $\left(5 \times 10^{5} / \mathrm{ml}\right)$ and volumes to construct the model of cell-cell direct contact. Used Millicell Culture Plate Inserts of $0.4 \mu \mathrm{m}$ pore size to construct the model of cell-cell indirect contact, MSCs grew on its lower chamber and HUVECs grew on its lower chamber with the same density $\left(5 \times 10^{5} / \mathrm{ml}\right)$ and volumes.

2. The VEGF levels of experimental group (culture media of co-cultured for $48 \mathrm{~h}$ ) and control group (MSCs and HUVECs's mixed culture media after them were cultured alone for $48 \mathrm{~h}$ ) were confirmed using ELISA assays. Co-cultured for 5 days. The MSCs's expression of fetal liver kinase-1(Flk-1), von Willebrand factor (vWF) and the ingestible ability of Dil labelled acetylated lowdensity lipoprotein (Dil-ac-LDL) were analysed by immunofluorescence staining, at the same time the changes of ultrastructure of cell-cell contact co-cultured cells were observed by transmission electron microscopy.

\section{Results}

1. MSC were negative expressed Flk-1 and vWF protein; HUVECs typically expressed the endothelial characteristics of vWF. The cells of cell-cell direct contact were observed under inverted phase contrast microscope and showed two kinds of cells gradually fused, the cells gradually close and difficult to distinguish.

2. the VEGF content of Experimental groups (559.55 \pm 66.19$) \mathrm{pg} / \mathrm{ml}$ were significantly higher than control groups (373.98 $\pm 57.28) \mathrm{pg} / \mathrm{ml}(\mathrm{p}<0.05, \mathrm{n}=3)$. Identified by immunofluorescence techniques, in direct contact co-culture system, by excited of different light from the laser scanning confocal microscope, fluorescence of MSCs nuclear labelled DAPI showed blue fluorescence, some MSCs of blue nuclear expressed Flk-1 protein and showed red fluorescence, then part of the Flk-1 positive MSCs expressed vWF protein and showed green fluorescence; besides, some MSCs could phagocytose Dil-ac-LDL. However, in indirect contact co-culture system, MSCs were negative expressed Flk-1 and vWF protein.

Observed ultrastructure of cell-cell contact co-cultured cells under transmission electron microscopy, we found karyoplasmic ratio of undifferentiated MSCs was big (karyoplasmic ratio >1.5); the nuclear forms was irregular, had notch and had 2-3 nucleoli inside nucleus; scarce and less developed organelles in cytoplasm. However, proportion of Mature HUVECs nucleoplasm was small (karyoplasmic ratio<0.5), HUVECs had regular nuclear shape, prominent nucleoli, abundant cytoplasmic organelles. MSCs during differentiation was observed the reduced karyoplasmic ratio and relatively abundant organelles, and electronic gap junctions between HUVECs and MSCs's the local of membrane; besides, cell fusion be seen.

\section{Conclusions}

1. MSCs can differentiate into endothelial cells through cell-cell contact by direct co-culture with HUVECs and have phagocytic ability of Dil-ac-LDL.

2. The mechanism that the cell-cell contact co-culture system can induce MSCs differentiation into endothelial cells may associated with the following factors:

(1) Cell-cell direct contact promote cell autocrine and paracrine VEGF increase significantly, involving in promoting the differentiation of MSCs. (2) Exist of cell fusion takes part in this process. (3).The form gap junction of intercellular communication between HUVECs and MSCs's the local of membrane. 